\title{
Mechanical properties of carbon nanotube fibres: St Venant's principle at the limit and the role of imperfections
}

\author{
Thurid S. Gspann ${ }^{\mathrm{a}^{*}}$, Nicola Montinaro ${ }^{\mathrm{a}, \mathrm{b}}$, Antonio Pantano ${ }^{\mathrm{b}}$, James A. Elliott ${ }^{\mathrm{a}}$, \\ Alan H. Windle $\mathrm{a}^{*}$ \\ a. Department of Materials Science and Metallurgy, University of Cambridge, 27 Charles Babbage Road, \\ Cambridge, UK, CB3 OFS \\ b. Dipartimento di Ingegneria Chimica, Gestionale, Informatica e Meccanica, Università degli Studi di \\ Palermo, Viale delle Scienze, 90128, Palermo, Italy
}

\begin{abstract}
Carbon nanotube (CNT) fibres, especially if perfect in terms of purity and alignment, are of extreme anisotropy. With their high axial strength but ready slippage between the CNTs, there is utmost difficulty in transferring the force applied uniformly. Finite element analysis is used to predict the stress distribution in CNT fibres loaded by grips attached to their surface, along with the resulting tensile stress-strain curves. This study demonstrates that in accordance with St Venant's principle very considerable lengthto-diameter ratios $\left(\sim 10^{3}\right)$ are required before the stress becomes uniform across the fibre, even at low strains. It is proposed that lack of perfect orientation and presence of carbonaceous material between bundles greatly enhances the stress transfer, thus increasing the load it can carry before failing by shear. It is suggested that a very high strength batch of fibres previously observed experimentally had an unusually high concentration of internal particles, meaning that the pressure exerted by the grips would assist stress transfer between the layers. We conclude, that the strength of CNT fibres depends on the specific testing geometries and that imperfections, whether by virtue of less-than-perfect orientation or of embedded impurities, are actually major positive contributors to the observed strength.
\end{abstract}

* Corresponding authors. Thurid S. Gspann, Tel: +44 (0)1223 767 059, Email: tsg28@cam.ac.uk; Alan H. Windle, Tel: +44 (0)1223 334 321, Email: ahw1@cam.ac.uk 


\section{Introduction}

Carbon nanotubes (CNTs) have inspired scientists and the public imagination since their dramatic rise to prominence in the early 1990s, and generated considerable industrial interest for electrical, mechanical and sensing applications [1]. There remain however, challenges to realising their full potential in useful materials. One limitation is the fact that CNTs are of finite length and, although they may have an aspect ratio in excess of $10^{5}$, they are still short compared with macroscopic length scales. While the strength observed in the best CNT fibres is in the range typical for conventional high performance fibres, especially when expressed as a specific stress $(\mathrm{GPa} / \mathrm{density})$ they have many features in common with yarns. Indeed it is their yarn-like nature which gives the CNT fibre filaments much greater toughness than conventional rivals, making these materials, for example, much more tractable in weaving situations than ordinary carbon fibre. In this paper, we address the question as to why the strength seen in CNT fibres is (at least) 25 times smaller than that of the individual CNTs, and point to strategies for future improvement.

\subsection{Yarn science}

It is well-established [2] that an increase in filament length in staple yarns will lead to an increase in breaking load, tenacity (specific strength), and elongation at break. On the other hand there is no clear relationship between stiffness and filament length. Also, as with many other fibres, breaking strength decreases with increasing yarn diameter. Unquestionably, yarn strength depends not only on the strength of the filament elements and their length, but also on the initial alignment of these elements and the uniformity with which they carry the applied stress. There are also mechanical hysteresis effects which are possibly associated with this lack of uniformity [3].

Any yarn-like fibre will potentially show a transition in fracture mode from the fibre pulling apart by shear between the filaments to one in which failure is due to the fracture of the filaments themselves. This transition will depend not only on the length of the filaments, but also on the friction between them as well as their individual tensile strength. In terms of CNT fibres there are two extreme scenarios: one for high interfilament friction, where the nanotube tensile strength would determine the yarn strength, and yarn-like properties such as the reported knot efficiency of $100 \%$ [4] would be lost and traded for brittleness, and the other for very low inter-filament friction, where the 
yarn might be viewed as formed from a lubricant material, and one would hardly expect it to be strong. In yarn science, the control of friction is a key objective [5], so we review here what is known about friction forces between CNT layers.

\subsection{Friction in graphene, graphite, and CNT assemblies}

That the very low friction between the graphene layers in graphite is associated with the comparatively weak interlayer interactions is long-established [6]. It is clear that the interlayer shear strength in graphite does not comply with friction in the conventional, macroscopic sense. Studies of few layer graphene and highly oriented pyrolytic graphite (HOPG) [7] suggest that the friction force is not only very low but tends to be independent of the normal force, and thus does not obey the first of Amontons' laws of friction. Studies on individual graphene layers are more difficult as the probe produces a large local elastic deformation (its own moving indentation) which complicates the interpretation [8], [9]. However, the frictional force is reported to be proportional to the true area of contact [7]. The dependence of the sliding friction on the sliding velocity is not yet established beyond doubt. One study [8] suggests no velocity dependence while another suggests a positive relationship between velocity and shear drag force [9].

Modelling of the shear force between graphene layers demonstrates both the observed very low friction levels, and the effect of commensurate stacking (crystallographic register) in increasing friction [10], [11]. It is reasonable to assume that packing between CNTs, or between layers of multiwall CNTs is normally incommensurate. Measurement made in pulling out inner cylinders of multiwall CNTs [12] or single wall tubes from a stack of similar tubes [13], demonstrate very clearly the additional, and reversible, contribution to the sliding forces associated with the creation of new surfaces, a force independent of overlap length [14]. Friction measurements on parallel CNTs in contact again indicate very low values of shear force which are independent of overlap length within measurement accuracy. However, if the CNTs are at all defective or contaminated, the shear force, now largely proportional to overlap length, is increased very considerably [15].

The contact area dependence for CNTs in fibres has been investigated by Zhang et al. [16], who found that the collapse of CNTs led to increases in inter-tube friction by a factor between 1.5 and 4. According to their calculations, un-collapsed CNTs would have to be at least five times longer than collapsed tubes to lead to the same specific 
strength. This agrees well with the observations [17] and also with molecular dynamics simulations [18], [19], [20] that high tensile strength fibres are seen in structures comprised mainly of collapsed tubes. In summary, the evidence is that the shear strength between CNTs in contact will be very low as long as there is no surface contamination, and that this shear strength will not increase significantly with normal pressure, with the result that in a tensile test, the pressure of the grips will not assist inter tube stress transfer over the gripped length.

\subsection{Structure of CNT fibres}

The structure of yarn-like CNT fibres consists of bundles of typically 50 nanotubes which have a high level of mutual local alignment [21]. The CNTs have a very high aspect ratio $\left(>10^{4}\right)$, as estimated by the small number of tube ends observed in the TEM. The CNT bundles form a continuous network, with the bundles branching and entangling but seldom terminating, at least as far as is observable by SEM and TEM. The structure is depicted in the electron micrographs in Figure 1A and 1B. The diameter of the single wall CNTs in the bundles is estimated from the positions of the Radial Breathing Mode (RBM) peaks in the Raman spectrum to be $0.9-1.5 \mathrm{~nm}$ [22]. Figure 1B also shows carbonaceous contamination on the external surfaces of the bundles. While the origin and composition of this extraneous material is not yet clear, it is known from the friction studies between CNTs that it adds significantly to the inter-bundle shear strength.
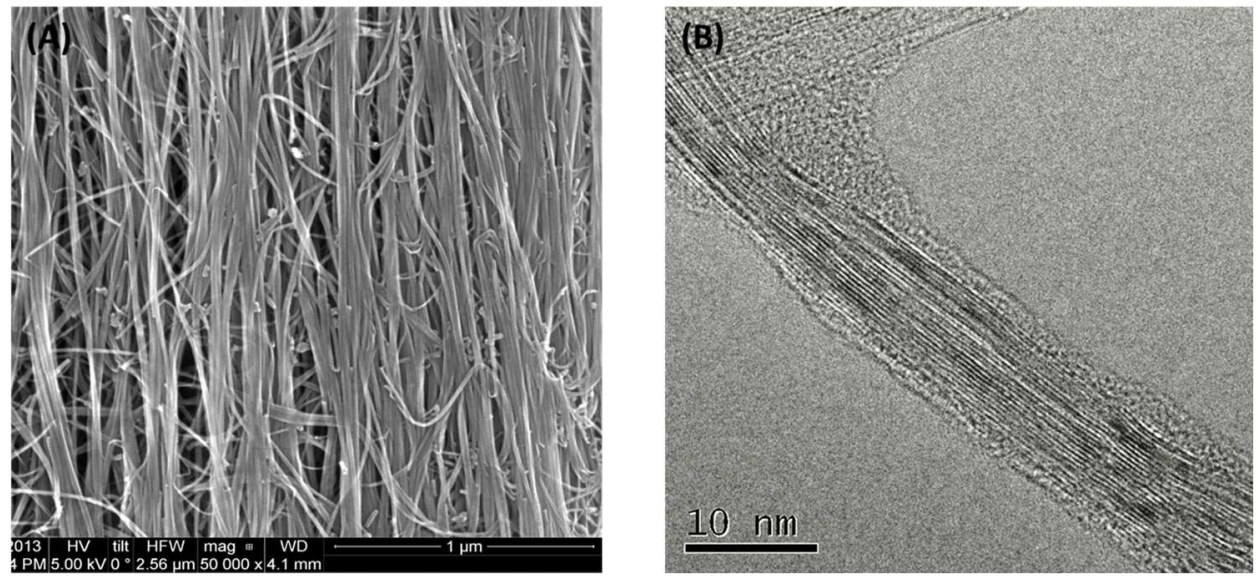

Figure 1: A) SEM of the surface of a condensed CNT fibre. The CNT bundles are aligned with the fibre axis (vertical). The branching provides a measure of interconnectivity between the CNT bundles. B) TEM of a SWCNT bundle evenly coated with co-synthesised carbonaceous material, branching at the lower right into smaller bundles. According to SEM and TEM, we treat CNT bundles as the basic elements forming the fibre, rather than individual CNTs. 
In an earlier report [23], a calculation was made of the strength of a bundle of CNTs. Given a literature-based estimate of the shear strength between pristine CNTs of $50 \mathrm{kPa}$, and an experimentally-based indication that the length of the individual CNTs in the fibre is of the order of $1 \mathrm{~mm}$ [17], and that the fibre under consideration then consisted of collapsed double wall CNTs, Vilatela et al. calculated the strength of a bundle to be $3.5 \mathrm{GPa} /$ specific gravity ( $\equiv \mathrm{N} /$ tex , where tex is a unit of the fibre linear density). A corresponding estimate for a sample of single wall CNTs, $1.5 \mathrm{~nm}$ in diameter, is of the order of $4 \mathrm{~N} /$ tex ( [23], equation 2). It should be pointed out, however, that these estimates of bundle strength assumed that the applied stress had been perfectly transmitted to all of the CNTs comprising the bundle.

For conventional twisted yarns such as cotton or wool, the twist imparted to the yarn means that tensile stress causes the fibre to compact on itself under tension and considerably increase the inter-filament friction enabling good tensile properties to be achieved with filaments (staple fibre) which have aspect ratios as low as $1000-5000$ ( [24] , p.252). However, even though any compressive forces arising from twist in CNT fibres are not predicted to increase the friction as a result of increasing the normal force - unlike conventional yarns - twisting will nevertheless increase the compaction of the fibre and thus the inter-bundle contact area. There is also a second order effect in that compressive stress may increase the polygonisation at inter-CNT contacts thus further increasing the contact area [25].

We have mentioned the possible role of contamination in enhancing the inter-bundle shear strength. However, there are other ways in which such enhancement may be achieved. Most of these involve the introduction of covalent cross links between neighbouring graphene layers whether by irradiation [26] or thermal means. It should be noted, however, that the introduction of crosslinks does increase the defect density in CNTs themselves thus compromising the intrinsic strength of the tubes. Further, any such improvement in the strength and stiffness of CNT fibres is likely to come at the expense of their outstanding toughness in bending, which itself is a consequence of comparatively easy inter-tube sliding.

It is clear that for yarn-like CNT fibres, the shear strength between the nanotubes or their bundles is many orders of magnitude less than the strength of the tubes in axial tension. We now address the central question of this paper. With such a low shear strength, how successful is a tensile test in transmitting the stress from the grips to the 
whole cross-section of the fibre, so that all the CNTs take a proportionate share of the load? Or to put it another way, to what extent does St Venant's principle ${ }^{1}$ apply to specimens as anisotropic as ours? To answer this question, we have modelled the tensile test explicitly using Finite Element Analysis (FEA).

\section{Strategy for the finite element model}

\subsection{Strategy}

In order to increase the computational efficiency of the FEA procedure, we have reduced our CNT fibre to a two-dimensional model of sliding layers. The steps in the argument are illustrated in Figure 2.

The element type in each layer which we consider to be critical is the bundle, as the lateral contact within a bundle is almost perfect (Figure 1B), while not as continuous between the bundles (Figure 1A). The mode of fracture of the fibres also appears microscopically to involve a sliding apart of bundles [4]. However, as will become clear in Figure 3, the choice between bundles or CNTs as key sliding elements will not greatly influence the predicted results and would be seen as the difference between the '100' and '1000 layer' curves.

On the assumption of axial symmetry of the fibre clamped around its circumference, each concentric layer of component filaments in a three-dimensional model is deemed to be uniformly stressed. The load transfer from each concentric layer of a cylinder model to the next can be assumed to be proportional to the contact area, and thus the radius, while the stress level in the layer into which the force has been transmitted will be proportional to the force divided by the cross-sectional area, again proportional to the radius. The scaling is thus the same as for a simple stack of sheets, as verified by satisfactory agreement between the mechanical predictions of the two models when run

\footnotetext{
${ }^{1}$ St. Venant's principle states that “.... the difference between the effects of two different but statically equivalent loads becomes very small at sufficiently large distances from load." [36,34], or in other words the distribution of the stress over a cross section in a fibre equalises with increasing distance from the test clamps in an axial mechanical test. There are caveats in the literature von Mises [37] that in absolutely strict terms St Venant's principle only applies to long, thin structures with infinite aspect ratio. However, Timoshenko et al. [35] suggest a rule of thumb that the length over which the locally applied load decays is about the diameter, although this would obviously only apply to isotropic material.
} 
in FEA (SOM Figure S 1) using a mesh of 4-node linear quadrilateral elements in Abaqus - called "CPS4". The 2D planar model is the basis for the following study.

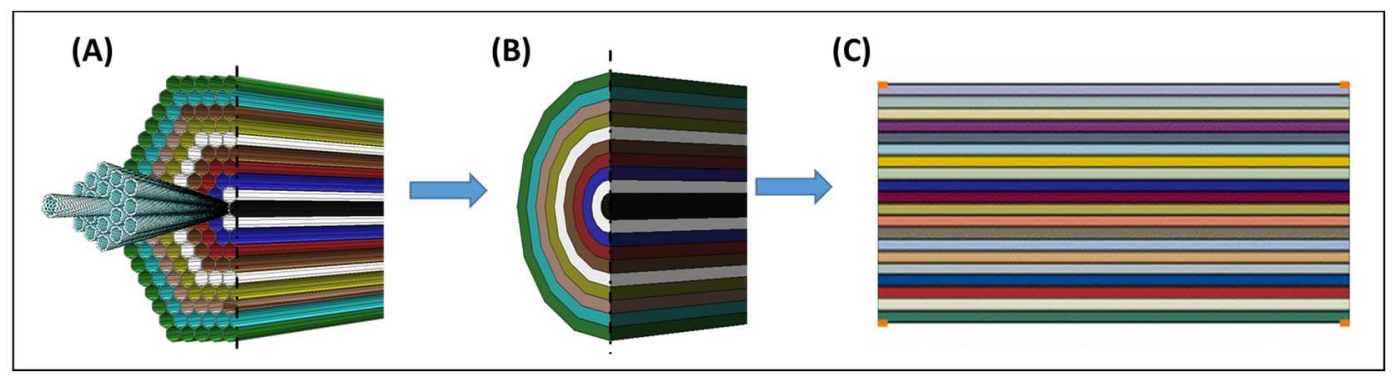

Figure 2: The model of the fibre was derived by fragmenting the fibre into sliding elements representing CNT bundles. In order to minimise computational time, the modelling was performed on a planar, two dimensional analogue of the fibre. From (A) a model of hexagonal elements, each depicting bundles of CNTs with perfect internal contact, the fibre structure is first reduced to $(B)$ a model of concentric cylinders, under the assumption that the stress within each cylindrical shell of bundles is constant. Now the structure can now be further reduced to one of planar layers (C) as the shear force transmitted from layer to layer is proportional to the circumference. SOM Figure S1 shows the comparison of the stress distribution for the cylindrical and plane sheet model.

\subsection{Interface modeling and choice of parametric values.}

While our major objective was to assess the behaviour of the model under stress and its capability to qualitatively predict effects of changing mechanical testing parameters, we also aimed to parameterise it as realistically as possible. Initially, the inter-bundle shear strength was set to be $50 \mathrm{kPa}$, as discussed above, in line with a range of literature values and used previously in a simple analytical model of CNT bundle strength [23]. For the model, the slip behaviour was divided into elastic shear of the layers using the published value of shear modulus of $1.1 \mathrm{GPa}$ [27] and plastic slip between the layers. The computational routine also provides for elastic shear in the interface between the layers up to a maximum value of $50 \mathrm{kPa}$ reached at the onset of plastic slip at $50 \mathrm{~nm}$. The model also requires a value for the axial stiffness of each layer (CNT bundle), which was set at $250 \mathrm{GPa}$. While this value is already far less than the ideal value for in-plane graphite of $1109 \mathrm{GPa}$ [28], CNT bundles usually show significantly lower values than $250 \mathrm{GPa}$. The reason for these lower values may reside in the difficulty of transferring the load to the CNT elements in the centre of the sample. As the presented model uses bundles as the basic element, neglecting internal sliding inside the bundles, we chose values for the intrinsic stiffness of a nanotube bundle which were in line with experimental data where the transfer through shear appears to be much more effective 
as a result of cross-linking by heating or radiation between the nanotubes and nanotube bundles [29].

The geometry of the model is shown in Figure 3, with the dimensions defined. We have a starting "standard model", where the grip length $l_{G}$ is $2 \mathrm{~mm}$ and the gauge length is $20 \mathrm{~mm}$. In the following we have changed the gauge length and grip length of the model relative to this standard. The model thickness was taken at $10 \mu \mathrm{m}$ in all cases; a value in the middle range of our experimental results for fibre diameter. The grips were assumed to be perfectly attached to the outer layer of the model.
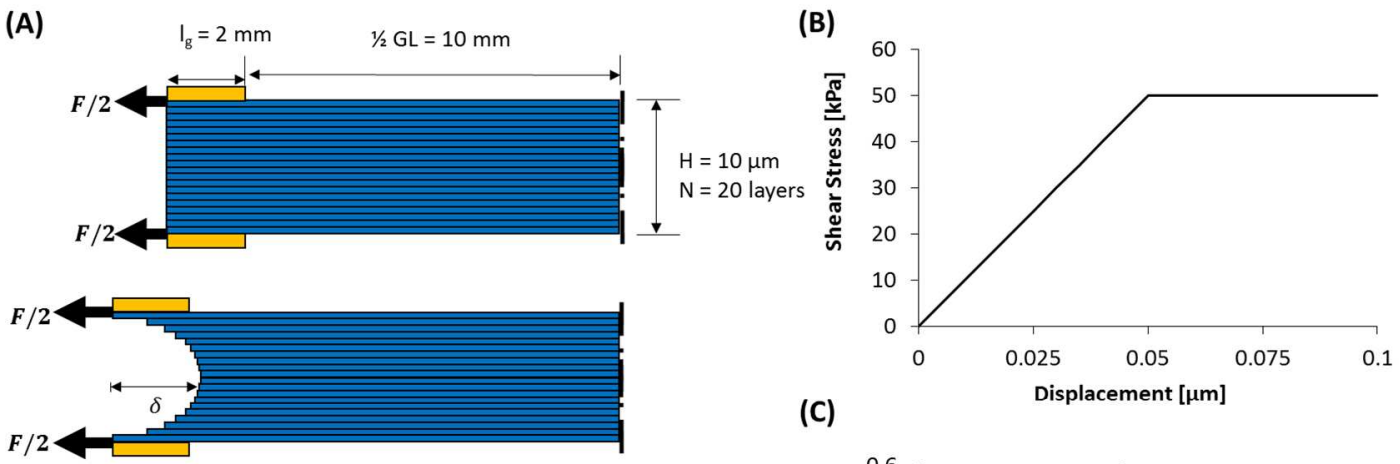

(c)

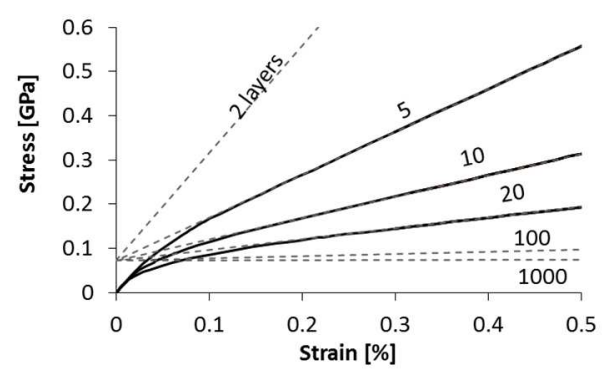

Figure 3: A) Schematic of a standard model comprised of perfectly aligned layers, with gauge length $(G L)=20 \mathrm{~mm}$, grip length $\left(l_{g}\right)=2 \mathrm{~mm}$, fibre diameter $(H)=10 \mu \mathrm{m}$, and number of layers $(N)=2 .($ for clarity laterally compressed $)$ $B)$ The shear stress between the layers was defined as non-linear, increasing until $\tau_{f}$ reached $50 \mathrm{kPa}$ at a slip displacement of $50 \mathrm{~nm}$ before sliding occurs. C) Stress-strain curves for the standard model comprised of 5, 10 or 20 layers. Extrapolating the trend lines (dotted) from when linearity is reached back to ordinate assigns an intercept stress of 72 MPa for which the shear stress is overcome and sliding commences. The trend lines are shown for the three values of $N$, and for $N=2,100$ and 1000 .

\section{Results}

\subsection{Model testing}

We applied the finite element analysis to the standard model (as defined in Figure 3A), varying the number of layers making up its thickness of $H$. If we consider the basic structural element of our fibre to be an individual nanotube, then, for the two dimensional analogue considered here, we would need up to 10,000 layers. This would 
be prohibitive in terms of computing resources, so we modelled fewer layers with the aim to extrapolating to a more realistic number. In Figure 3C, the stress-strain curves are shown for the standard model comprised of various layer numbers. The plots show an initial slope slightly less than the prescribed axial stiffness for layers of $250 \mathrm{GPa}$. The slope then begins to decrease, with the plots eventually assuming linearity at a much lower slope that is dependent on the number of elements into which the model is divided. The initial slope corresponds to the situation before sliding has started to occur. Once sliding is initiated between the outside layers and the layers immediately underneath, the slope begins to decrease, eventually approaching linearity at a slope corresponding to the axial stiffness of the outside layers only. Beyond this point, sliding is occurring both within the grips and along the gauge length, so that any further increase in load corresponds to the stretching of the outer layer only which is only a fraction $1 / N$ of the total model thickness, where $N$ is the number of layers. When $N$ is large, this second slope will therefore be very small. In Figure $3 \mathrm{C}$, it is seen that the second portions of the curves approach lines which all extrapolate back to the same point on the stress axis. This point is at $72 \mathrm{MPa}$, which is the axial stress at which sliding is established, the friction stress $\tau_{\mathrm{f}}$ being exceeded. The sliding occurs between the outer layer and that adjacent to it over the complete length of the sample both within the grips and over gauge length.

For this most simple of model geometries, it is possible to confirm the salient aspects of the FEA-predicted stress-strain curves by a simple calculation. This exercise is carried out here to check that the FEA model predictions, which include stress-strain curves and stress distributions both along and across the model, are in the expected range. Assuming that the sample is of unit 'depth' in the third dimension, the axial stress expressed as the friction force over the sample thickness, where sliding is assumed to occur over the full sample length (including that inside the grips) can be approximated by:

$$
\sigma=\tau_{\mathrm{F}}\left(\mathrm{GL} / 2+\alpha \mathrm{l}_{\mathrm{G}}\right) / \mathrm{H}
$$

where $G L$ is the gauge length, $l_{G}$ is the grip length and $H$ is the fibre diameter. The factor $\alpha$ can be assumed to be unity where there is no pressure effect enhancing friction 
within the grips. For the dimensions of the "standard model" as described in Figure 3, and a friction stress $\tau_{\mathrm{F}}$, of $50 \mathrm{kPa}$, the axial stress from this most simple of equations is $60 \mathrm{MPa}$ which is close to the FEA predictions of $72 \mathrm{MPa}$.

\subsection{Cross-sectional stress distribution.}

For the standard FEA model, which predicted the curves of Figure 3C, the stress distributions at different distances from the grips in a $20 \mathrm{~mm}$ gauge length sample are shown in Figure 4. At low strains (dotted line) before plastic shear between layers commences, the stress distribution is relatively uniform across the model thickness. However, once sliding is well established ( $0.2 \%$ strain (solid line), c.f. Figure 4$)$, the stress concentration in the outer layer is very apparent. These plots show that $\mathrm{St}$ Venant's principle takes a considerable distance to become manifest in such a highly anisotropic material. Indeed, for $0.2 \%$ strain, it is some 780 fibre diameters before the stress at the fibre core even reaches one half of that at the surface! 


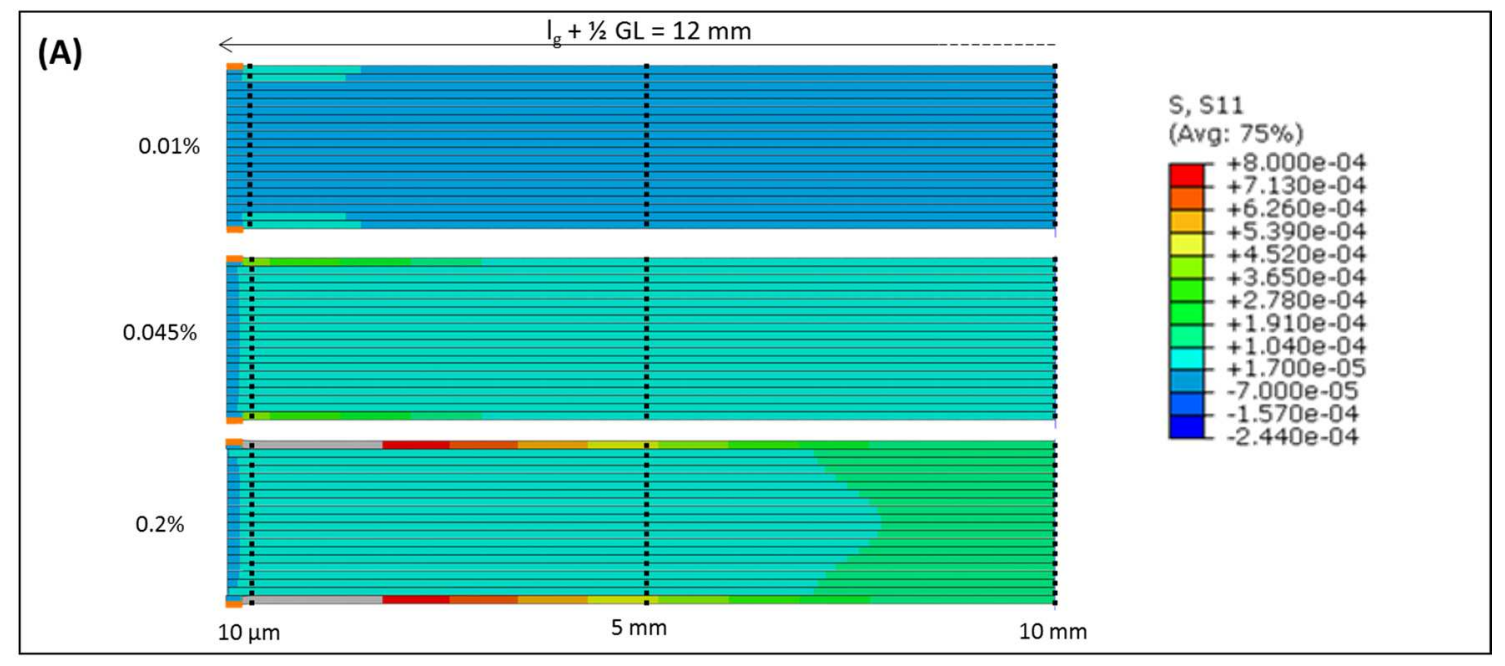

(B)
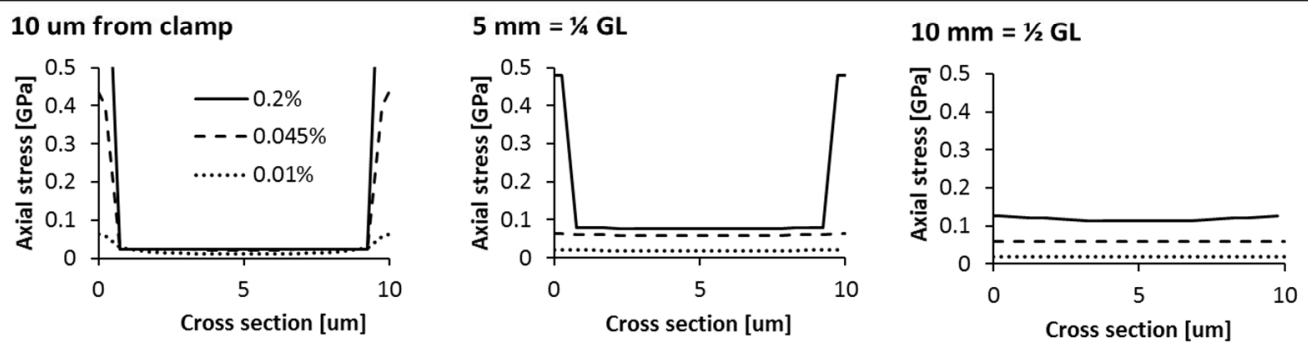

Figure 4: The colour plots of the model demonstrate the stress distribution for 0.01\%, $0.045 \%$ and $0.2 \%$ strain. For better clarity the model is shown laterally compressed by a factor of 200. The clamps are compressed by a further factor of 10. In agreement with St. Venant's principle, the stress, which is initially concentrated only in the outer layers touching the clamp surface, gets transferred inside the fibre core over a long distance. The cross-sectional stress distribution graphs for 3 positions - near the clamps (distance $10 \mu \mathrm{m}$ ), at quarter gauge length and half gauge length - illustrate that at a small strain of only $0.2 \%$ the stress at half gauge length (a factor of 1000 diameters away from the clamps!) has only barely reached equilibrium.

It is not surprising that if the gauge length of the model is reduced to $1 \mathrm{~mm}$, there is little transference of the stress from the surface layers to the material as a whole, presenting an even starker challenge to the validity of the tensile test for these parameters. The stress distributions of Figure 5A attest to the total inapplicability of St Venant's principle when testing fibres of extreme anisotropy using normal laboratory testing geometries. The predicted stress-strain curve of Figure 5C shows an intercept (sliding) stress of $22 \mathrm{MPa}$, compared with a value from the simplistic model above of $12.5 \mathrm{MPa}$. It is interesting to note that the initial modulus appears much less than the axial value of $250 \mathrm{GPa}$ chosen for each layer. The reason is that at such short gauge lengths, the elastic shear deformation, incorporated into the FEA model as a 
shear modulus, will account for a greater proportion of the grip displacement relative to the axial extension of the elements.
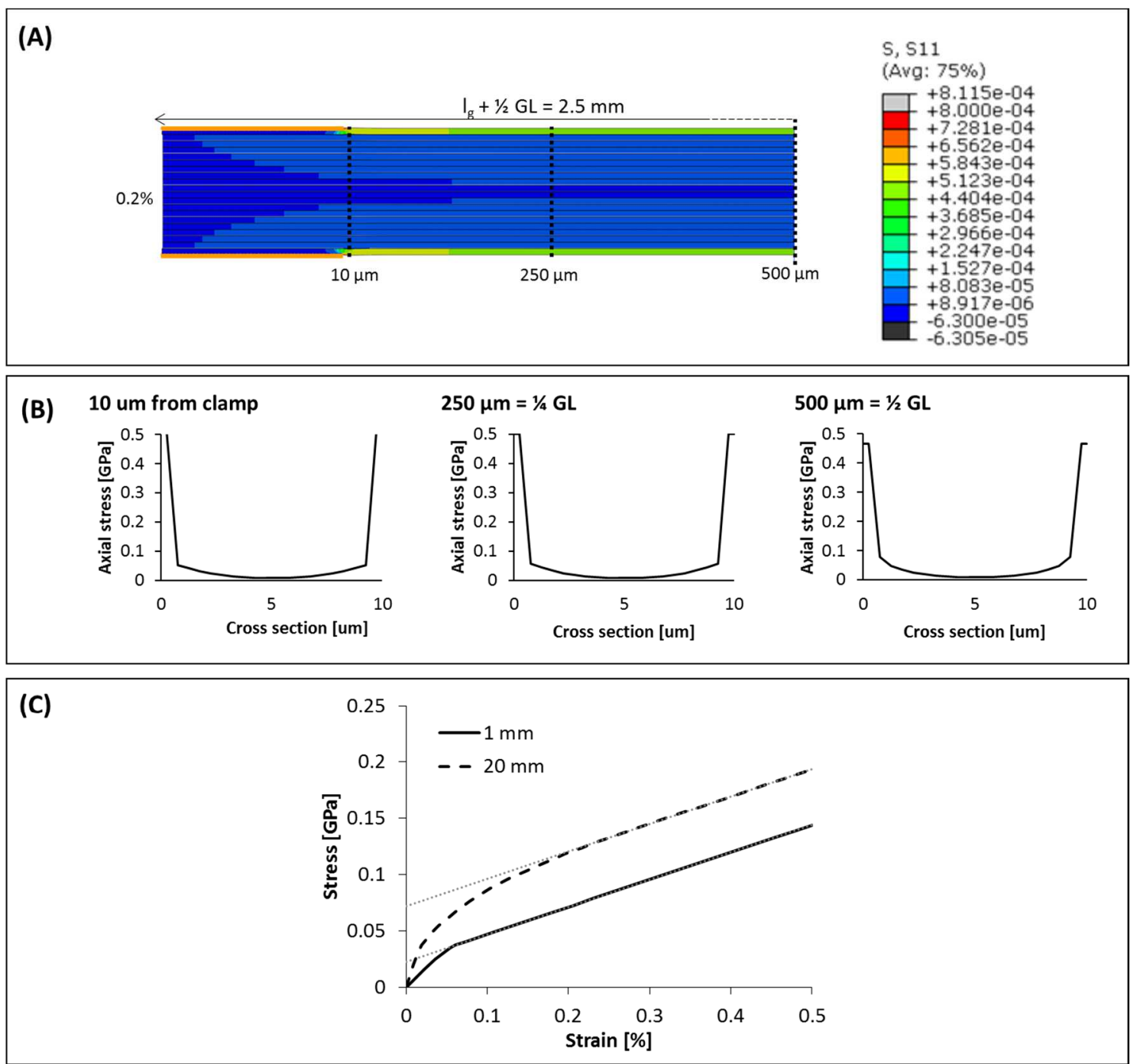

Figure 5: (A) The colour plot (aspect ratio distortion of 15) and graphs of cross-sectional stress distribution in a short gauge length model $(G L=1 \mathrm{~mm})$, under otherwise standard conditions, show that even for low strains crosssectional stress does not reach equilibration. (B) The predicted stress-strain curves for gauge length $20 \mathrm{~mm}$ and $1 \mathrm{~mm}$ show a decrease of the initial modulus at short gauge length, and of the intercept stress to only $22 \mathrm{MPa}$, indicating that the elastic shear deformation commencing in the grips dominates over shear in the free gauge length.

For the situation when the gauge length is held constant but the ratio $\mathrm{lg}_{\mathrm{g}} / \mathrm{GL}$ increased, the influence on the stress-strain curves is particularly marked. From equation 1 we would expect to see a pronounced increase in intercept stress. The FEA outputs of Figure 6 bear this out. The stress distribution (SOM 2) shows that at the grip exit point more stress has been transferred to the inner layers for the longer grips are, and that the 
stress across the whole samples becomes essentially uniform over a greater proportion of the gauge length.

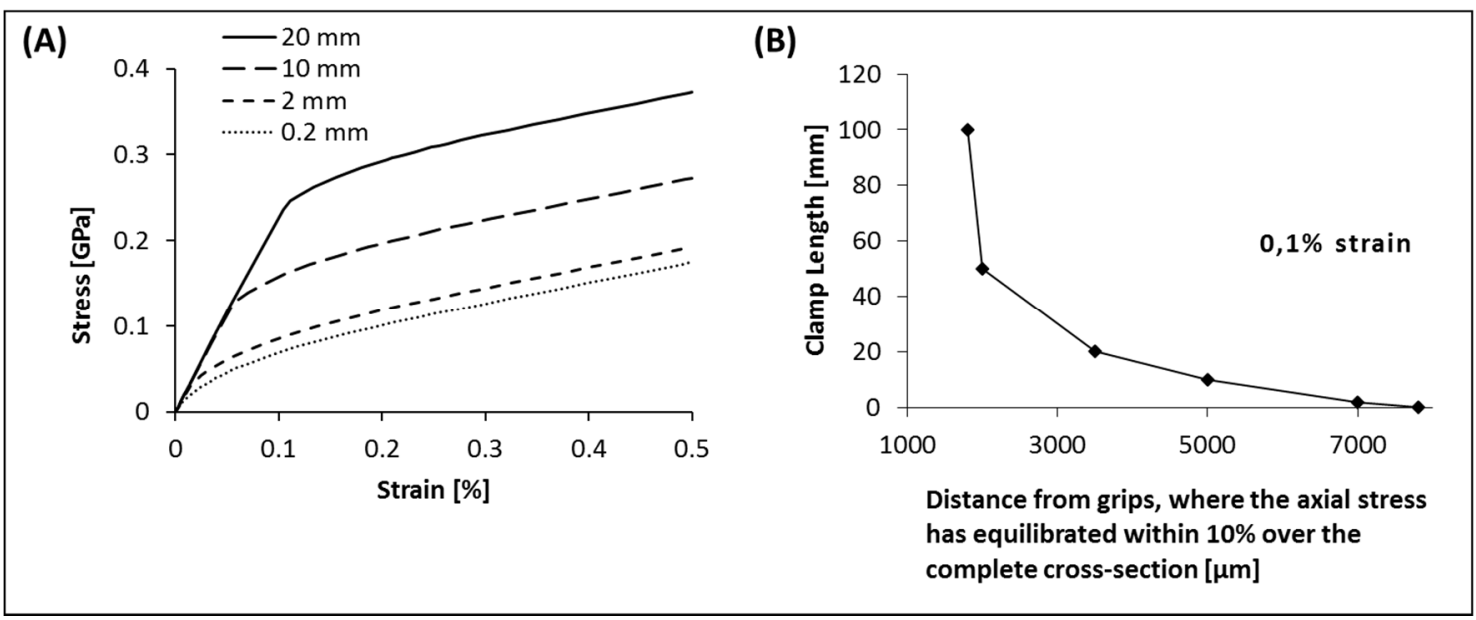

Figure 6: A) Increase of grip length leads to increase of intercept stress, occurring at a stress only slightly below the maximum possible load. (B) Distance from the grips where the variation of the axial stress distribution has reduced to $10 \%$ depending on the clamp length.

The axial stress for complete interlayer sliding (the intercept stress) was found to depend linearly on the value used in FEA for the friction stress, $\tau_{\mathrm{F}}$ (Figure 7 ). Increased friction stress also meant that the through-thickness stress distribution become more uniform at distances much closer to the grips - a prediction also in line with the simple model above. For a value of $500 \mathrm{kPa}$, ten times that of the standard model, the variation in the through-thickness stress distribution was reduced to $10 \%$ at only $1.65 \mathrm{~mm}$ from the grips. A degree of uniformity only reached at $9.5 \mathrm{~mm}$ for the $50 \mathrm{kPa}$ case. 
(A)
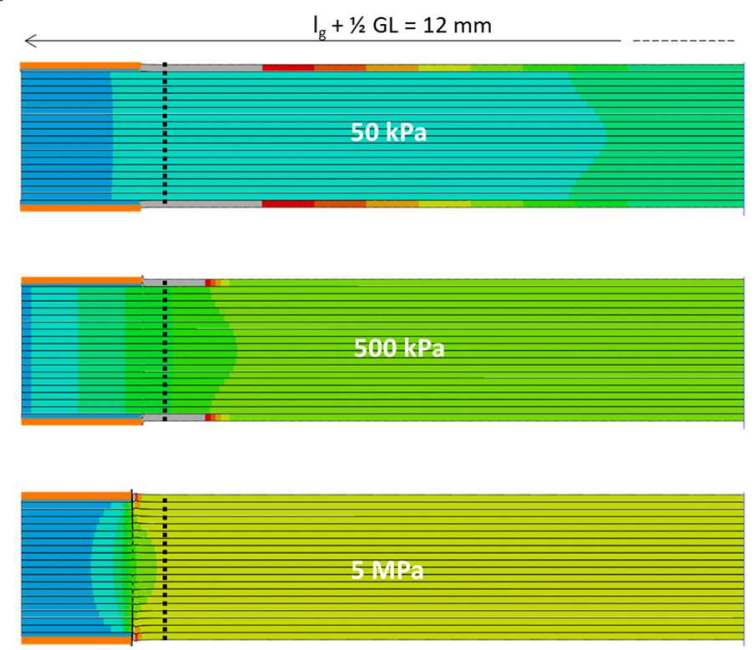

$1.5 \mathrm{~mm}$
(B)

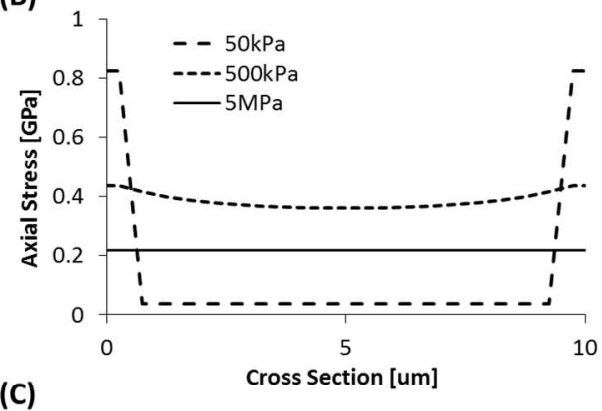

(C)

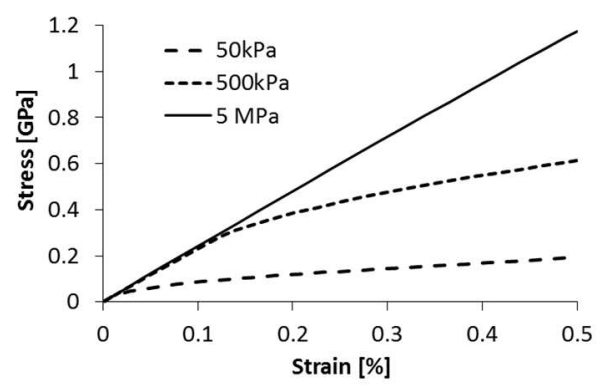

Figure 7: (A) Colour plots of stress distribution and (B) stress-distribution over the cross-section of the standard model for varied friction stresses ( $\left.\tau_{f}\right): 50 \mathrm{kPa}$ (standard), $500 \mathrm{kPa}$ and $5 \mathrm{MPa}$. The higher the friction stress is, the nearer to the clamps a uniform stress distribution is reached. (C) The predicted stress-strain curves showing the effect of increasing $\tau$.

\subsection{Summary of effect of geometric variables on the predicted sliding stress.}

The FEA model was run for a range of gauge length and grip lengths leading to the contour plot of predicted key stress parameters from the stress-strain curves shown as a function of geometry in Figure 8. The contour set (Figure 8A) show the predicted intercept values (c.f. Figure 3) which increase both with gauge length and grip length, although somewhat more rapidly with the latter. The intercept values predicted by the simple algebraic model (equation 1) generate a broadly similar contour map as shown in Figure S 3.

The contours in Figure 8B show the dependence of the yield tress at which the stressstrain curves first deviate from the initial elastic slope. This predicted stress value appears to be largely independent of the gauge length, being approximately directly proportional to the grip length. The data all correspond to the assumed friction stress, $\tau_{\mathrm{F},}$ of $50 \mathrm{kPa}$. The red circles represent experimental geometries which will be discussed below. 


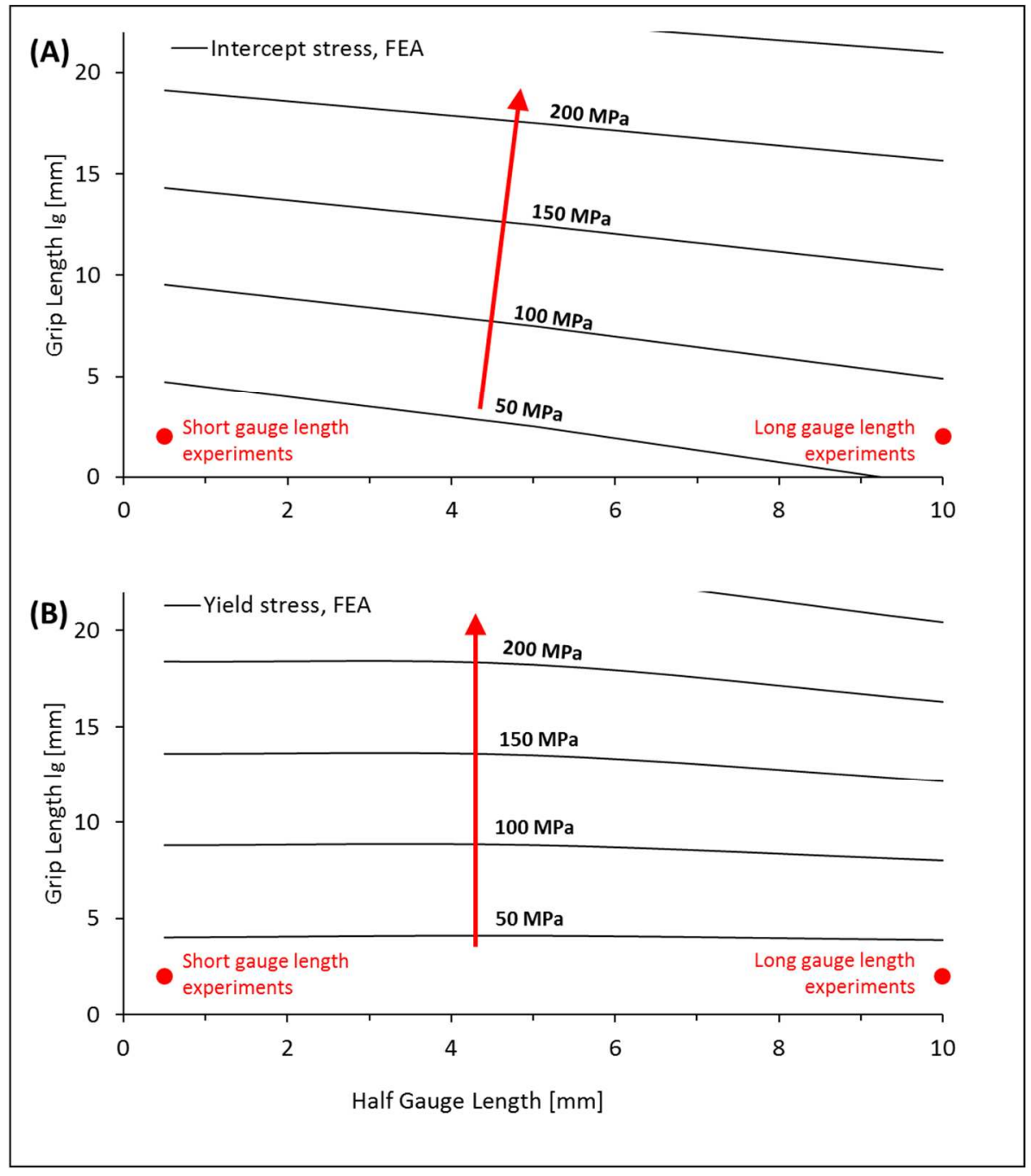

Figure 8: Contours of (A) the intercept (sliding) stress and (B) the yield stress according to the FEA model, as a function of grip length $l_{g}$ and half gauge length GL for a shear stress value $\tau_{f}=50 \mathrm{kPa}$. The typical experimental geometries are shown as red dots. While the intercept stress shows a distinct dependence on both grip length and gauge length, the yield stress appears to be independent of the gauge length up to grip lengths which are untypical for experimental test geometries. These plots are reproduced in SOM 3, where the point values of strength are also inserted in addition to the contours.

The model does not impose an upper limit of stress at which point the sample would break. There are however important indications from the predicted stress-strain curves. For situations where $N$ is large, and we identify layers of the model with CNT bundles, the curve will tend to horizontal at the intersection stress, with the implication that failure by sliding will occur without any increase in applied axial stress. The strain in the outer (top and bottom) layers will continue to increase until the failure strain is reached. To estimate this failure strain, given the assumed axial modulus of $250 \mathrm{GPa}$, one needs an estimate of bundle strength. We will take this to be $4 \mathrm{GPa} / \mathrm{density}$, after 
the modelling-based estimate of Vilatela et al [23], which is somewhat above the highest strength seen in fibres made routinely in our laboratory [22]. As we will be comparing these predictions with measurements made on fibres consisting of single wall CNTs, the parameters used in their equation are slightly different with a reduced polygonisation effect compensated by the fact that all the carbon is now on the surface of the single wall tube. In very simple terms, we would expect that the failure strain to be 4/250 expressed as a percentage, i.e. 1.6\%. However, the stress and thus axial strain in the outer layer is of course concentrated towards the grips, (as shown in Figure 3), so that the outer layer will break at a lower overall extension.

The stress at which the stress-strain curves first depart from the initial linearity we will refer to as the yield stress. For the standard sample, the yield stress is well below the intercept stress, although nearly coincident with it in the case of the short gauge length example (Figure 5B). The fact that this stress seems to depend only on the grip length (Figure 8) suggests that it corresponds to the point where slippage first occurs in the grips. Where this is below the intercept stress, the curved part of the stress-strain plot corresponds to the spreading of slippage along the gauge length. However, once slippage occurs between the outside layers and their immediate neighbours, the maximum stress that is translated into the central part of the sample within the grips is capped, so that further extension of the model will always increase the stress in the outer layers, especially adjacent in the grips, and thus failure by inter layer sliding will be assured. The FEA model predictions suggest that for the stress to be shared uniformly between the layers right up to their predicted axial breaking strength of $4 \mathrm{GPa}$ (for a friction stress of $50 \mathrm{kPa}$ ), the required grip length would be of the order of $420 \mathrm{~mm}$ ! Of course there are many assumptions in the model, but the results give an important indication that, under the geometries usually deployed for testing, the axial strength measured in samples with such high anisotropies in terms of the ratio of axialto-shear strength, may be very considerable underestimates.

\subsection{Experimental values.}

The two red circles plotted on Figure 8, correspond to two testing geometries for which data are available (SOM 6). In the context of the model predictions, Type A2 and B (SOM 6, and described in detail in [22]) show very much higher strengths than the contours would suggest, and also show no significant dependence on gauge length. 
However, for each sample the fracture mode appears to involve the pulling apart of the bundle elements, i.e. failure by shear.

At first sight, it may be simply a matter that the value taken for the friction stress is too low by at least an order of magnitude. Running the model with different values of $\tau_{\mathrm{F}}$, shows that this parameter behaves essentially as a scaling factor to the predictions (Figure 3), so that both the yield stress and the intercept stress are proportional to its value. However, bearing in mind that the experimentally measured strength is the same at both gauge lengths (SOM Figure S 6), we note that the predicted values of intercept stress are dependent on gauge length, with the FEA model giving a reduction by a factor of three on reducing the gauge length from the standard $20 \mathrm{~mm}$ to $1 \mathrm{~mm}$ (a reduction in aspect ratio from 2000 to 100). By contrast however, the yield stress (as defined by loss of initial linearity) is not sensitive to the gauge length, as it corresponds to the onset of slip within the grips.

\subsection{Applicability of the model to the actual fibres}

Before concluding that $50 \mathrm{kPa}$ is far too low for the shear failure stress inside CNT fibres, it is important to consider the ways in which an actual fibre is different from the simple model employed so far. By virtue of the FEA model, it is also possible to address some of this increasing complexity. The real fibre appears to be less than ideal in several respects:

(a) The orientation of the CNT bundles being less than perfectly axial, with the additional factor that there may be topological entanglements despite the high degree of alignment. (3.5.1)

(b) The CNT bundles having effective lengths less than that of the gauge length and the total grip length. (3.5.2)

(c) The existence of other extraneous material in the fibre samples which may increase the interfacial shear strength between the bundles. (3.5.3)

(d) The inclusion of particulate defects, often associated with residual catalyst for fibres drawn direct from a floating catalyst reactor. (3.5.4)

\subsubsection{Orientation.}

The axial orientation of the CNT bundles is not perfect as may be seen in Figure 2A. It has been quantified by small angle X-ray diffraction, through a measurement of the azimuthal smearing of the fibre streak [30], where the width at half magnitude was of 
the order of $\pm 6^{\circ}$, a value that is qualitatively compatible with the image. The exact relationship between the average quality of axial alignment given by scattering measurements and the degree to which a given set of CNTs or bundles, wanders from attachment surface in the grips to transfer stress through the fibre thickness will depend on the detailed model. However, a first indication can be achieved by assuming that the CNT bundles undergo a random walk through the fibre thickness with distance along the fibre. Taking the mis-orientation to be $\pm 6^{\circ}$, then a bundle of mean diameter $50 \mathrm{~nm}$ will be displaced laterally with respect to the fibre axis by an average distance of around $0.5 \mu \mathrm{m}\left(50 \mathrm{~nm} / \tan 6^{\circ}\right)$.

Let us now consider a CNT bundle at the edge of the fibre, whose lateral displacement, $x$, undergoes an unbiased random walk as a function of its position along the axis of the fibre, $t^{\prime}$. In this case, the root-mean-squared value of its lateral displacement from edge of fibre will be $\left\langle x^{2}\right\rangle^{1 / 2}=a N^{1 / 2}$, where $a$ is the smallest length of each 'jump' (equal to $50 \mathrm{~nm}$, the bundle diameter) and $N$ is the number of lateral jumps made per unit displacement along the fibre axis (equal to the displacement along fibre, $t$, divided by $\left.50 \mathrm{~nm} / \tan 6^{\circ}=0.5 \mu \mathrm{m}\right)$. Hence, $\left\langle x^{2}\right\rangle^{1 / 2}=7 \times 10^{-5}\left(t^{\prime}\right)^{1 / 2}$. The distance along fibre at which the mean position of bundles that were originally situated at edge of fibre are now located in middle of fibre (diameter $10 \mu \mathrm{m}$ ) is then $t^{\prime}=\left(\frac{5 \times 10^{-6}}{7 \times 10^{-5}}\right)^{2}=5 \mathrm{~mm}$.

While these calculations are very approximate, and apply to the two dimensional model of the FEA simulations rather than the three dimensional fibres, the important conclusion is that the observed mis-orientation implies that a significant fraction of the fibres which were in contact with the grips would have transmitted the applied stress to the centre of the sample over a distance of the same order as the grip lengths used. The question arises as to whether the transfer of stress due to the wandering of less than ideally aligned bundles can be seen simply as an enhancement of the inter bundle friction stress. It is tempting to say that the underestimate of observed strengths arising from the perfectly aligned models by an order of magnitude or more, can be corrected simply by an increase in $\tau_{\mathrm{F}}$ to some higher 'effective' value. In order to test this, an FEA model has been generated in which the elements exchange positions at random, although any model in which the same layer in contact with the grip at one end returned to contact the grip on the other fibre end was eliminated. The stress distribution in the 
region of one of the grips is shown in Figure 9A. The points at which the layers exchange positions (swap) are shown by the short vertical lines, and sometimes short gaps. In this model there are two such points under the top grip (note that the whole model is scaled down horizontally by a factor of 200 , and the region in the grips by a further factor of 10 . Note how the stress within the two layers which contact the upper grip is high and how the stress is transferred further into the sample with further swaps.

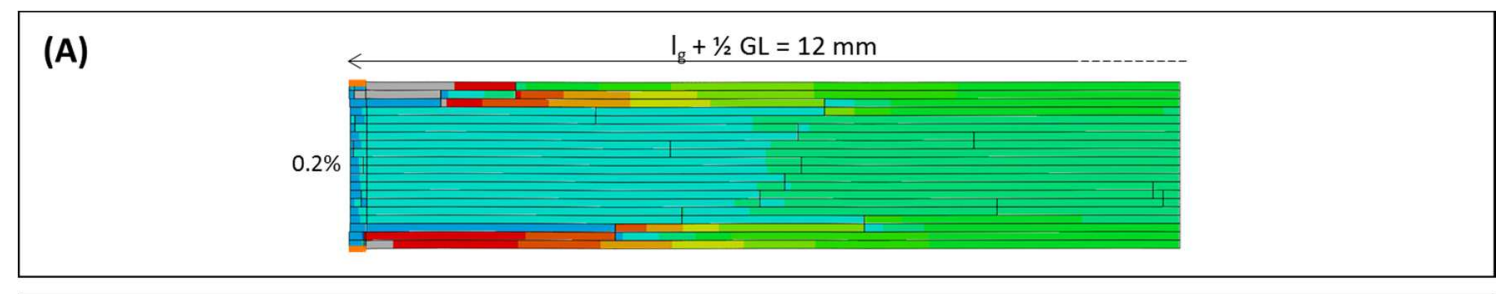

(B)

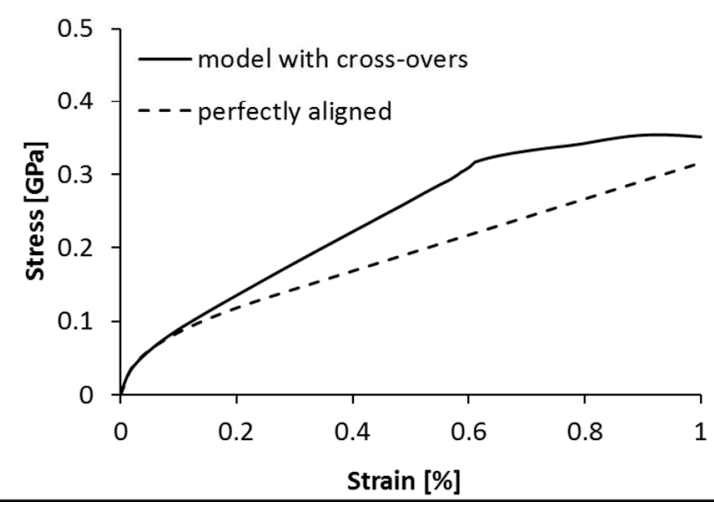

Figure 9: (A) The colour plot for a model in which the layers change places at random positions including one swap in the grip touching layers, though no layer is allowed to be attached to the grip at both ends. Other than the introduction of cross-overs the model corresponds to the long gauge length standard, laterally shrunk by 200 in the gauge length, and by a further 10 in the grips. (B) The comparison of stress-strain curves for the swap model with the perfectly aligned model show an increased slope after the yield stress, corresponding to now 2 layers touching each clamp and transmitting elastic shear to 2 neighbouring layers each. While for the simple model, the pull-out stress will correspond to the intercept stress, for the model including cross-overs it would correspond to the upper, nearly horizontal stress.

Figure 9B shows the predicted stress-strain curve compared with that for the perfectly aligned model. In each case the initial departure from linearity, i.e. the yield stress, corresponds to slipping within the material in the gripped region, although, in the case of the swap model, it will be under the first two layers, each of which is attached to the grip at some point - see detail in Figure 9A. As described above, the slope after the yield stress of the perfect model is due to the elastic stretching of the surface element attached to both grips, and this slope will decrease with increasing number of layers becoming nearly horizontal for layer thickness equivalent to bundle diameter. However, 
there are no such doubly-connected layers in the cross-over model, so the slope after the yield will represent the stretching and slipping within the sample of each of the two layers attached to each grip up to the point at which the sliding stress, $\tau_{\mathrm{F}}$, is reached over their entire length. The cross-over model will fail by pull out of layers which are attached to the grip at one end, pulling right through the gauge length and the opposite gripped region, while failure for the perfect model will occur only when the top layer reaches its failure strain in tension. The start of this failure process for the cross-over model shows on the stress strain curve (Figure 9B) as the onset of the initial horizontal portion. If the number of layers attached to each grip component remains at two, then one would expect the second near-linear slope of the predicted stress strain curve to be reduced as the axial stress on each grip-attached layer will be increased. For large $N$, the pull out stress would be the intercept stress for the perfectly aligned model. But for the "cross-over" model it would correspond to the stress of the upper, near horizontal portion of the stress strain curve: a stress about 5 times higher. The reason for this higher stress can be understood in general terms, as there are now two layers attached to each grip component, and each of these layers, once it becomes buried in the fibre, experiences frictional retardation on both top and bottom surfaces. There is thus a factor of 4 expected on the basis of this most simple argument. Finally, if the mis-orientation angle is to be preserved for a model with increasing values of $N$, then one might expect that a greater proportion of layers would have the advantage of being in contact with, and thus being pulled directly by the grips. In this case, one might expect that at high $N$, the slope of the second near-linear portion of the stress-strain curve would be mainly independent of $N$. However, the failure stress in shear would increase linearly with $N$. We thus conclude that the fact that the CNT bundles are not perfectly aligned compensates for the inefficiency of stress transfer in shear between them, and is a much more efficient mechanism of transferring the surface stress in the grips towards the centre of the gauge length. Our FEA models suggests that lack of perfect orientation is a major contributor to the fact that the observed strength is at least an order of magnitude higher than that predicted by the standard (perfectly aligned) model.

\subsubsection{Samples with a characteristic length less than the gauge length.}

The CNT fibre is certainly yarn-like, and by analogy with staple yarns such as those of cotton, one would expect the strength to increase at short gauge lengths which are less than the length of the component, staple filaments. If such terminations are present in 
CNT fibre, they are difficult to identify. They could include CNT ends, or bundle terminations or indeed reversing loops of either. The microstructure of the fibre (Figure 1) gives only very limited evidence of any type of termination. Another type of defect may be a region parallel with the fibre axis in which there is limited or no exchange of branching bundles giving an interface which would be particularly weak in shear. In either case, bundle termination defects or defects in the shear plane, one would expect the strength of shorter tensile specimens to be larger than longer ones, in much the same way as any sample with random defects will be weaker as the length and thus probability of a defect increases, in accordance with a Weibull-type analysis.

Even in the standard (perfect) model, the bundles are of limited length, i.e. the gauge length plus both grip lengths. A very simple approach would be to say that the influence of increasing grip and gauge lengths in increasing stress transfer into the fibre will be limited in each case to the characteristic length of the components of the microstructure. Hence the increase in the predicted axial strength of the models both with gauge length and grip length, and the increase in 'yield stress' with grip length, as summarised in Figure 8 , would be terminated once the characteristic length of the fibre elements is exceeded. While an increase in the effective value of $\tau_{F}$ as discussed in the previous section may at least in part account for the much higher experimental strengths compared with the model predictions, it will only scale the predicted values, and will not explain why experimentally the longer gauge length geometry does not give an increased strength as predicted by the model. It is possible however, that the easier sliding of the shorter gauge length sample is being compensated by the increased probability of bundles traversing the whole sample length without a defect causing a termination (further discussion in SOM 4).

\subsubsection{Carbonaceous impurity deposit.}

While there is no doubt that the lack of perfect orientation is an effective stress transfer mechanism from the gripped surface towards the centre of the tensile test sample, it raises another issue: namely that the contact length between adjacent CNT bundles is compromised (see Figure 1A). The regions of lack of longitudinal contact can also be seen as crack-like defects parallel to the fibre axis as envisaged in Figure 1. These axial 'cracks', which account for some 50\% of the volume of the fibre, while reducing the effectiveness of stress transfer would also be the sites for the initiation of failure in shear, and of course do not feature in the perfect model. Examination of the structure 
of the fibre in the TEM, typically shows a carbonaceous deposit on the surface of the bundles but not within the bundles where nanotube-nanotube contact appears to be perfect. On some occasions the deposit contains precipitated iron particles (SOM Figure S 5). It appears that this carbonaceous material is the result of synthesis reactions as the aerogel moves out of the hot zone of reactor [21], [22], making the reasonable assumption that not all of the carbon has been converted into CNTs. We suggest that this material is effective in mitigating the deleterious effect of the longitudinal cracklike defects. We have previously observed [31] that the back diffusion of a monomer into the fibre, followed by its UV initiated polymerization could double the observed strength. There have also been reports of carbonaceous deposit seen on the bundles in direct spun fibres, which have been associated with an influence on mechanical properties. In particular Espinosa and co-workers [32] have indicated that these deposits could be the origin of the hysteresis observed in stress-strain curves on repeated cycling. In a recent paper [22], we have reported mechanical data on fibres synthesised with a much-reduced level of particulate impurities and with a high proportion of single wall tubes, which show strengths approaching $2 \mathrm{~N} /$ tex. These fibres also show a marked carbonaceous deposit on the bundles, which we associate with the good axial properties. The exact properties, amount and identity of this carbon-rich deposit appear to depend strongly on the precursors used in the CNT synthesis, and are the subject of on-going experiments. These deposits may prove to be a method of controlling and enhancing the mechanical properties of the fibres. We also note here also the observation [33], that CNT yarns infiltrated with pyrocarbons via chemical vapour infiltration show an increased strength and stiffness by factors of 2.6 and 6.5 , respectively.

In the search for further analogies, we mention that natural fibrous materials are often stabilised and strengthened through inter-fibrillar binding material. For example, cellulosic fibrils in plant material are surrounded by hemicellulose and lignin, or collagen fibrils of the animal kingdom surrounded by hydroxyapatite within the cell. By forming a concave coating, the branching points of the fibres become softened, which could be considered as starting points for cracks.

\subsubsection{Particulate impurities.}

The presence of internal particles, particularly if large relative to the fibre diameter, could be expected to reduce the bundle contact and impair mechanical properties of CNT fibres, both strength and stiffness. Furthermore, with stress being expressed as 
$\mathrm{N} /$ tex ( $\equiv \mathrm{GPa} / \mathrm{SG}$ ), additional weight that did not contribute mechanically would further compromise properties. On the other hand, catalyst residues, often with an over-coating of carbon, typically of the size $4-7 \mathrm{~nm}$ though up to $20 \mathrm{~nm}$, do not appear to disrupt the bundle contact significantly. The samples containing particles tend to show an enhanced strength at short gauge lengths $(\leq 2 \mathrm{~mm})$, an observation totally in accord with the yarn-like nature of the individual as-spun fibre. As a result of recent process developments [22], we have been able to decrease the amount of co-synthesised impurities and residual catalyst leading to a uniform and dense fibre structure. However while such major reductions in particle content produced no increase in strength at long $(20 \mathrm{~mm})$ gauge length, surprisingly, the increase in strength apparent at shorter gauge lengths was lost for the cleaner samples. Though this can also be due to a decrease in characteristic length by decreased residence time in the reaction zone during synthesis, we are faced with the experimental conclusion that embedded particles do not reduce the measured strength of the fibres, although they do tend to reduce stiffness (further discussion in SOM S6).

Data previously published in 2007 [17] showed a strength peak of $\sim 1 \mathrm{~N} /$ tex, but also an increased probability of seeing strengths in the region of $5 \mathrm{~N} /$ tex as the gauge length was reduced. Subsequent testing of the same batch in our own laboratory again showed the secondary high strength peak in the distribution, though at a lower strength of 3.5 $4 \mathrm{~N} /$ tex. As made clear in the original publication, the result was peculiar to one particular batch of fibre and could not be reproduced in general. It did however generate an upper bench mark of what could be achieved in a CNT fibre, albeit at short gauge lengths. We have now re-examined with high resolution SEM a remaining sample from this original, extraordinary batch of fibre and observed that the fibre was exceptionally impure (SOM Figure S 5A and 5B). It showed all kinds of impurities discussed so far, from deformed MWCNT fibres [22], polymeric co-synthesised material [31], yet, the carbon encapsulated catalyst particles were in unusual abundance. Given the outcomes of the modelling reported above, we suggest that the all-but-beneficial influence of particles in short gauge length testing, may be due to the role of the particles within the gripped length, where they are compressed into the CNT bundles, and enhance the stress transfer from the grips into the body of the fibre, acting rather as 'internal sandpaper'. 


\section{Summary and Conclusions}

An assessment of the structure and properties of yarn-like CNT fibres has indicated a complex interplay between the properties expected of an ideal structure, where the major challenge would be to transfer the force applied by the grips throughout the whole thickness of the fibre. This consequence of the high axial strength of the CNT elements but the very low shear strength between them - a shear strength not enhanced by pressure within the gripped regions - means that for a perfectly aligned model, the stress transfer necessary for St. Venant's principle to apply would require extremely long total sample length. For the testing geometries used so far, the predicted fibre strength is more than an order of magnitude less than that observed experimentally, although failure in each case is a result of shear between the axially strong structural elements.

The lack of perfect alignment of the CNT bundles with the fibre axis provides a reason why the stress transfer from fibre surface in the grips across the gauge length may be enhanced, although a consequence of this lack of alignment between bundles is that there are axial regions of much reduced interfacial shear strength. We suggest that the deposit of amorphous carbonaceous material onto the surface of the bundles which acts as an inter-bundle 'adhesive' mitigates the weakening effect of the longitudinal defects. In samples with high particulate content, there is no decrease in specific strength, but actually an enhancement of strength at short gauge lengths. While it is possible that the particles compromise the degree of alignment thus improving stress transfer across the fibre, it is also possible that they enable grip pressure to enhance the stress transfer within the grips, as a type of 'internal sandpaper'.

The work has shown that the strength achievable in CNT fibres results from a complex interplay not only of the properties of the nanotubes themselves, but defects in their organisation and the presence of extraneous material some of which is formed during the cool-down stage of the process. The study suggests particular experiments which can be made in as a result of adjusting process parameters, especially with regard to the post hot zone deposition of carbonaceous and catalyst material, and measuring the influence of this impurity material on mechanical properties. The understanding the various roles of defects in affecting mechanical properties, opens up the prospect of tailoring these novel fibrous materials to provide a wide range of properties to order. 


\section{Acknowledgements:}

The authors are grateful to USN ONR GLOBAL for the provision of funding under award number N62909-14-1-N200. Any opinions, findings, and conclusions or recommendations expressed in this material are those of the author(s) and do not necessarily reflect the views of the Office of Naval Research.

Thurid Gspann is particularly grateful to Dr Patrick Kiley for the help with the molecular representation of the CNT bundle in figure 2 .

\section{References}

[1] Volder MF, Tawfick SH, Baughman RH, Hart AJ. Carbon nanotubes: present and future commercial applications, Science 2013, 339: 535-539.

[2] Morlier OW, Orr RS, Grant JN. The relation of length to other physical properties of cottong fibers, Text Res J 1951, 21(1): 6-9.

[3] Alen SR, Filippov AG, Farris RJ, Thomas EL. Structure-property relations in poly(p-phenylene benzobisthiazole) fibers, in The strength and stiffness of polymers, New York, Marcel Dekker Inc; 1983: 357-380.

[4] Vilatela JJ, Windle AH. Yarn-like carbon nanotube fibers, Adv Mater 2010, 22(44): 4959-4963.

[5] Broughton RM, Mogahzy YE, Hall DM. Mechanism of yarn failure, Text Res J 1992, 62(3): 131-134.

[6] Bragg WL. Introduction to crystal analysis, London. 1928: 64.

[7] Schwarz UD, Zwörner O, Köster P, Wiesendanger R. Quantitative analysis of the frictional properties of solid materials at low loads. I. Carbon compounds, Phys Rev B 1997, 56(11): 6987-6995.

[8] Zwörner O, Hölscher H, Schwarz UD, Wiesendanger R. The velocity dependence of frictional forces in point-contact friction, Appl Phys A 1997, 66: S263.

[9] Lee H, Lee N, Seo Y, Eom J, Lee S. Comparison of frictional forces on graphene and graphite, Nanotechnology 2009, 20: 325701 1-6. 
[10] Guo Y, Guo W, Chen C. Modifying atomic-scale friction between two graphene sheets: A molecular-force-field study, Phys Rev B 2007, 76: 155429 1-5.

[11] Shibuta Y, Elliott JA. Interaction between two sheets with a turbostratic orientational relationship, Chem Phys Lett 2011, 512(4-6): 146-150.

[12] Cumings J, Zettl A, Low-friction nanoscale linear bearing realized from multiwall carbon nanotubes, Science 2000, 289(5479): 602-604.

[13] Cornwall CF, Majure D, Haskins R, Lee NJ, Ebeling R, Maier R, et al. Critical carbon nanotube length in fibers, DoD HPCMP Users Group Conference, 2008.

[14] Akita S, Nakayama Y. Interlayer sliding force of individual multiwall carbon nanotubes, JJAP 2003, 42: 4830-4833.

[15] Suekane O, Nagataki A, Mori H, Nakayama Y. Static friction force of carbon nanotube surfaces, APEX 2008, 1: 064001 1-4.

[16] Zhang X, Li Q. Enhancement of friction between carbon nanotubes: An efficient strategy to strengthen fibers, ACS Nano 2010, 4(1): 312-316.

[17] Koziol K, Vilatela J, Moisala A, Motta M., Cunniff P., Sennett M, Windle A. High-performance carbon nanotube fiber, Science 2007, 318(5858): 1892-1895.

[18] Elliott JA, Sandler JKW, Windle AH, Young RJ, Shaffer MS. Collapse of singlewall carbon nanotubes is diameter dependent, Phys Rev Lett 2004, 92(9): 095501 $1-4$.

[19] Motta M, Moisala A, Kinloch IA, Windle AH. High performance fibres from 'dog bone' carbon nanotubes, Adv Mater 2007, 19(21): 3721-3726.

[20] Pugno NM, Elliott JA. Buckling of peapods, fullerenes and nanotubes, Physica E 2012, 44: 944-948.

[21] Li Y-L, Kinloch IA, Windle AH. Direct spinning of carbon nanotube fibres from chemical vapour deposition synthesis, Science 2004, 304(5668): 276-278.

[22] Gspann TS, Smail FR, Windle AH. Spinning of carbon nanotube fibres using the floating catalyst high temperature route: purity issues and the critical role of sulphur, RSC Faraday Discussions 2014, 173: 47-65.

[23] Vilatela JJ, Elliott JA, Windle AH. A model for the strength of yarn-like carbon nanotube fibers, ACS Nano 2011, 5(3): 1921-1927.

[24] Zhang X. Fiber and Polymer Science, DEStech Publications Inc; 2014. 
[25] Qian D, Liu WK, Ruoff RS. Load transfer mechanism in carbon nanotube ropes, Compos Sci Technol 2003, 63(11): 1561-1569.

[26] Peng B, Locascio M, Zapol P, Li S, Mielke SL, Schatz GC, HD Espinosa. Measurements of near-ultimate strength for multiwalled carbon nanotubes and irradiationinduced crosslinking improvements, Nat Nanotechnol 2008, 3: 626631.

[27] Salvetat J-P, Briggs GAD, Bonard J-M, Bacsa RR, Kulik A J, Stöckli T, et al. Elastic and shear moduli of single-walled carbon nanotube ropes, Phys Rev Lett 1999, 82(5): 944-947.

[28] Bosak K, Krisch M, Mohr M, Maultzsch J, Thomsen C. Elasticity of singlecrystalline graphite: inelastic x-ray scattering study, Phys Rev B 2007, 75: 153408 $1-4$.

[29] Filleter T, Bernal R, Li S, Espinosa HD. Ultrahigh strength and stiffness in crosslinked hierarchical carbon nanotube bundles, Adv Mater 2011, 23(25): 28552860.

[30] Qiu J, Terrones J, Vilatela JJ, Vickers ME, Elliott JA, Windle AH. Liquid infiltration into carbon nanotube fibers: effect on structure and electrical properties, ACS Nano 2013, 7: 8412-8422.

[31] Boncel S, Sundaram R, Windle A and Koziol K. Enhancement of the mechanical properties of directly spun CNT fibers by chemical treatment, ACS Nano 2011, 5(12): 9339-9344.

[32] Naraghi M, Filleter T, Moravsky A, Locascio M, Loutfy RO, Espinosa HD. A multiscale study of high performance double-walled nanotube polymer, ACS Nano 2010, 4(11): 6463-6476.

[33] Thiagarajan V, Wang X, Bradford PD, Zhu Y, Yuan F. Stabilizing carbon nanotube yarns using chemical vapor infiltration, Comp Sci Tech 2014, 90: 8287.

[34] Love AEH. A treatise on the mathematical theory of elasticity, Cambridge University Press; 1927.

[35] Timoshenko SP, Goodier JN. Theory of elasticity, 3rd ed, Tokio: McGraw-Hill. 1970: 39-59. 
[36] Saint-Venant AJ. Memoire sur la torsion des prismes, 14, Harvard University; 1853.

[37] Von Mises R, On Saint Venant's principle, Bull Amer Math Soc 1945, 51: 555562. 


\section{SOM}

\section{$1 \quad$ Cylindrical and stacked sheet model}

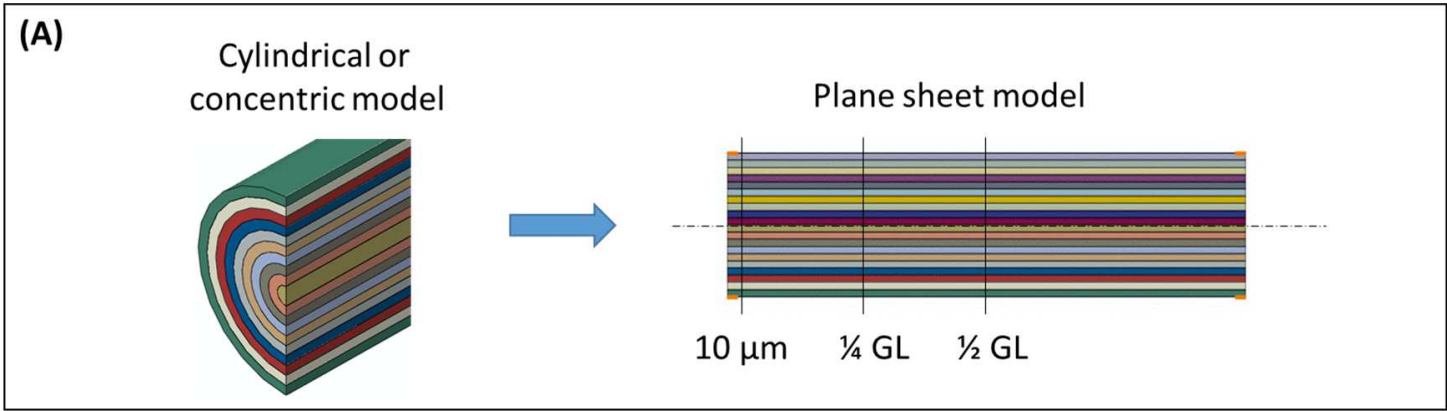

(B) 10 um from clamp
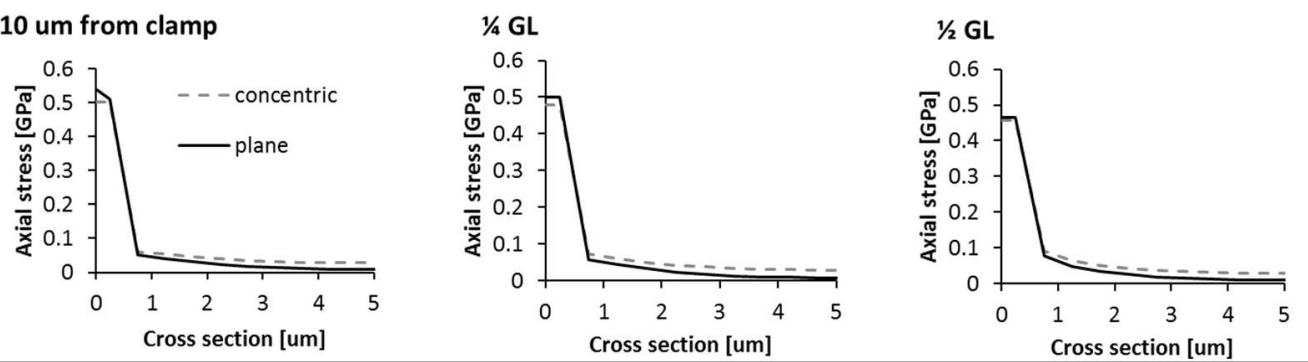

Figure S 1: Comparison of the stress distribution across the half cross section for the cylindrical model (dotted) and the plane sheet model (solid line) for short gauge length, otherwise standard (grip length $2 \mathrm{~mm}$, friction stress $\tau_{F}=$ $50 \mathrm{kPa}$, at $0.2 \%$ strain). 


\section{Clamp length effect}

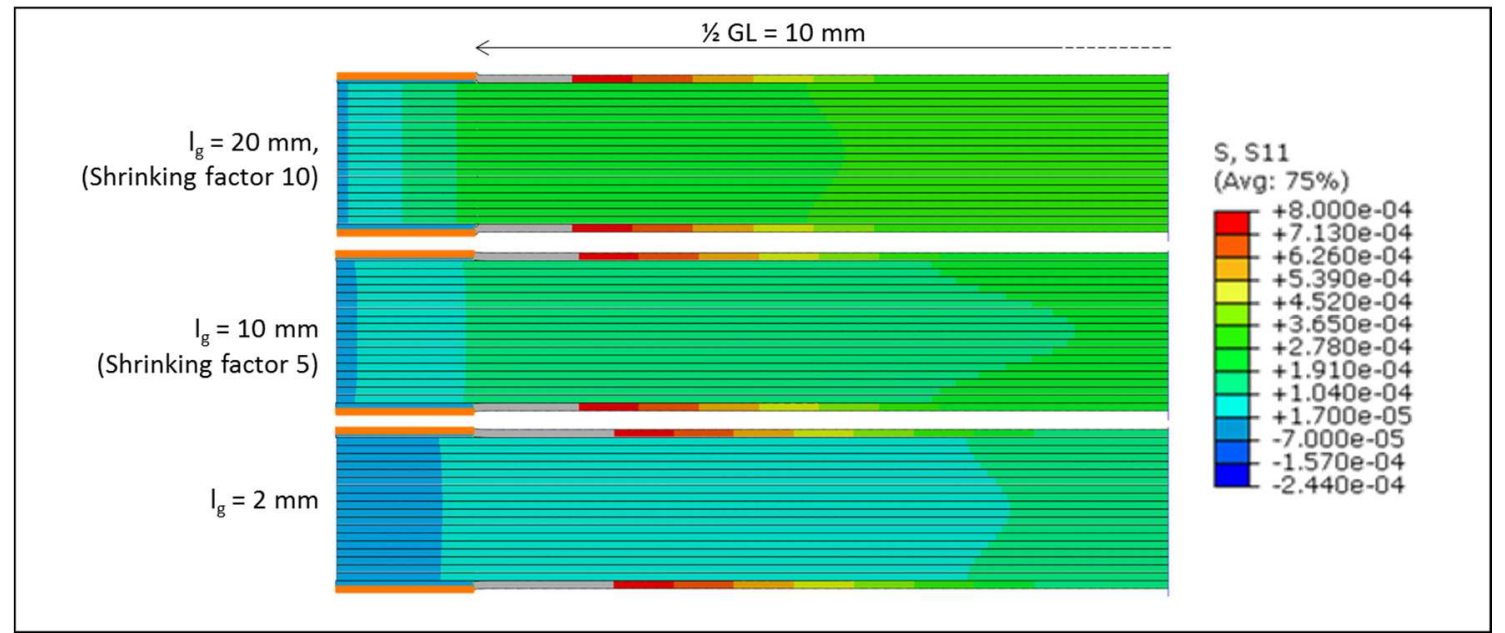

Figure S 2: (A) colour plots of axial stress distribution for grip length $2 \mathrm{~mm}, 10 \mathrm{~mm}, 20 \mathrm{~mm}$. For better comparability, the clamps have been scaled to the same length in the plots. 


\section{Contour plot}

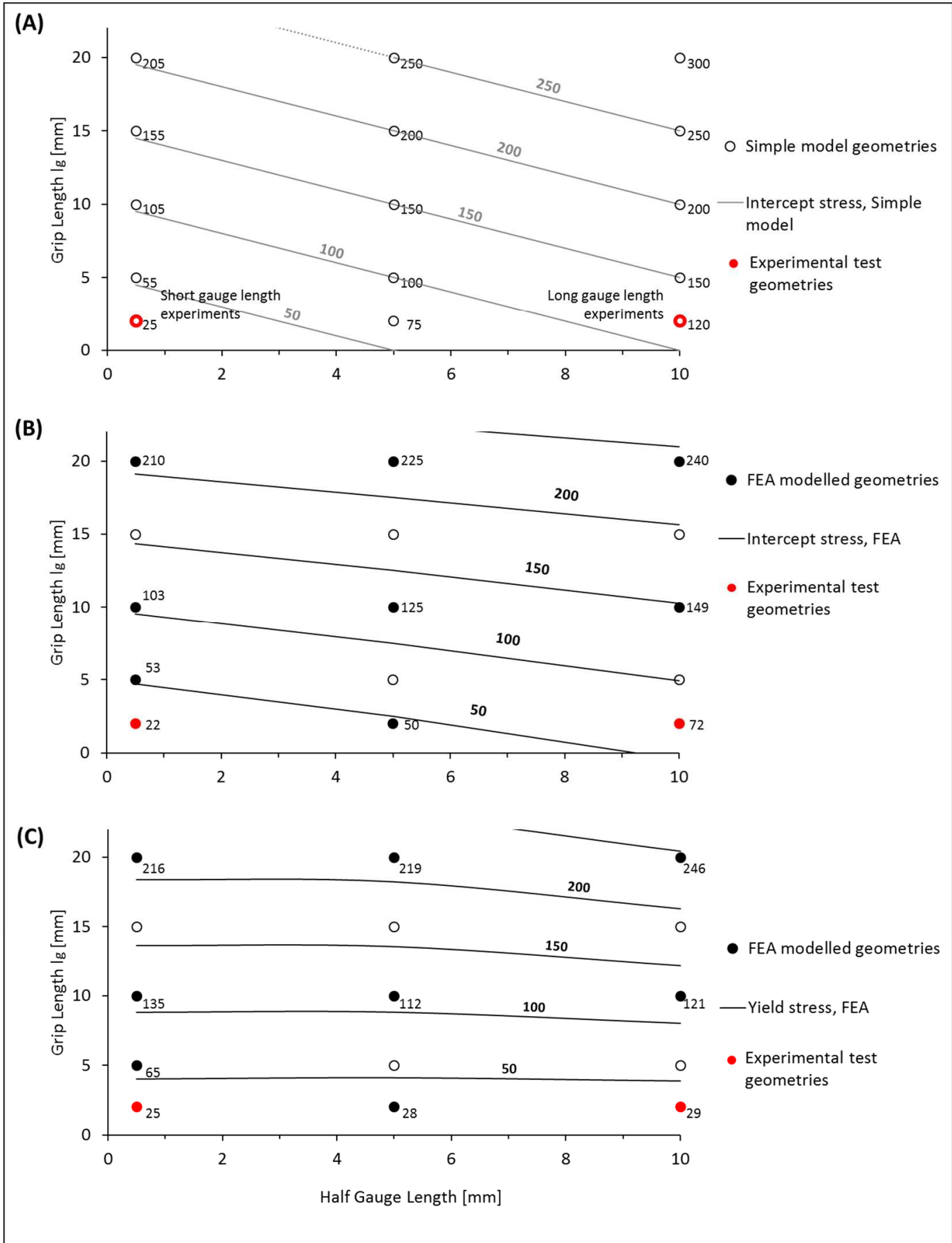

Figure S 3: Contour plots of the intercept stress predicted by (A) the algebraic model (equation 1), and (B) the FEA model, and $(C)$ the yield stress from the FEA model. The circles show the modelled geometries from the algebraic model (empty) and FEA (filled), labelled with the values in MPa from the respective model. The typical experimental test geometries - short and long gauge length - are shown as red points. Given the simplicity of the algebraic model, the values match the FEA derived values encouragingly well. While the intercept stresses depend on both gauge length and grip length, the yield stress appears to be largely independent of the gauge length, but directly proportional to the grip length. 


\section{$4 \quad$ Short gauge length and effect of ends}

Across all application fields, the influence of junctions and CNT ends is normally seen as detrimental. Electrically junctions are the main origin of resistance, mechanically CNT and bundle ends are the ultimate defect. Therefore we now study the effect of layer ends, by breaking the layers at random positions along the sample. Given the estimated CNT length of several hundred $\mu \mathrm{m}$, and a bundle length which is considerably longer but yet far off the long gauge length of $20 \mathrm{~mm}$, we can only expect to see a variation in number of layer ends for short gauge length tests. Hence the end study was executed only for GL $=1 \mathrm{~mm}$.

The result of non-continuous layers is firstly to have a stress-strain curve which is horizontal once sliding is established over the whole length of the sample. Figure S 4 shows the very different effect of layer ends for different friction stress scenarios. For low friction, as assumed in the standard model, we investigate 2 extreme cases. Firstly, if both outer layers touching the grips are unbroken, while the core layers are unbroken, the stress-strain curve overlaps exactly with the standard short gauge length curve. Secondly, if the outer layers are broken, but the core layers are all unbroken, the model is equivalent to the fracture state of the standard model, and after reaching the limit of elastic shear the curve is horizontal and the model fails by pull-out from within the gripped length.

For significantly higher friction, as could be assumed for a scenario with carbonaceous coating increasing the friction between bundles and additionally particulate deposits increasing the friction between the grips, the position of the unbroken layers is no longer crucial. 
Low friction $\tau \mathrm{F}$

Grip touching layers unbroken, inner layers broken

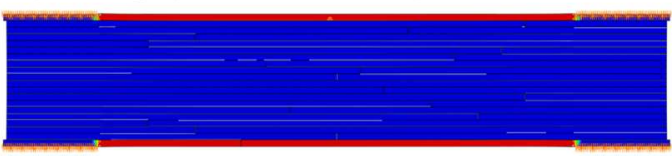

Grip touching layers broken, inner layers unbroken
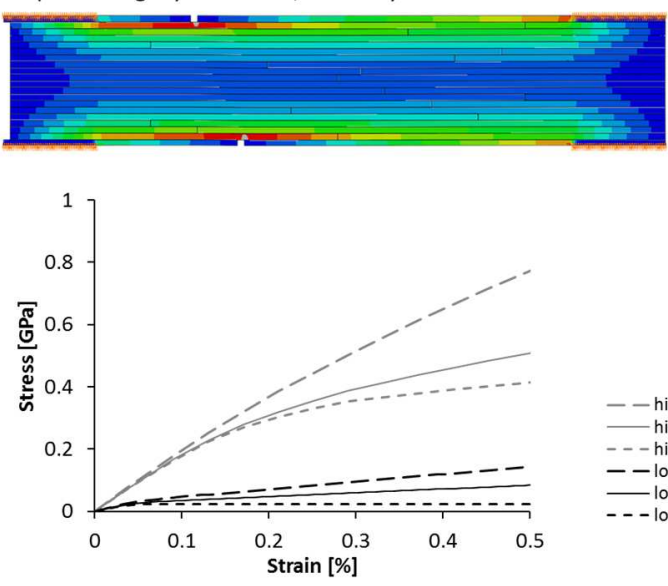

High friction $\tau_{\mathrm{F}}$

2 layers unbroken

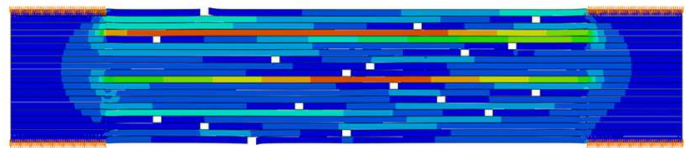

4 layers unbroken

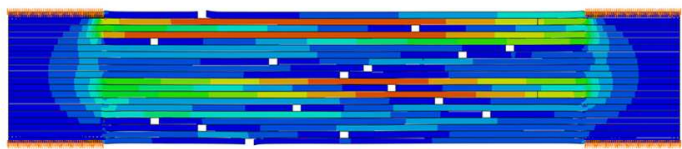

10 layers unbroken



- high friction, 10 gripped bundles high friction, 4 gripped bundles - - high friction, 2 gripped bundles - low friction, one outer layer unbroken - - - low friction, outer layers broken

Figure S 4: Study of the effect of layer ends in the gauge length. In case of low friction $\tau_{F}=50 \mathrm{kPa}$ (left) there are two extreme cases. Are the outer layers unbroken, they carry the total load equivalent to the simple standard model. Are the outer layers broken, however, the model equates the fracture state of the standard model and will fail by pull-out of the core layers within the grips. As soon as the outer layers are broken, the stress/strain curves show no difference whether any or all inner layers are unbroken. In case of increased friction (right) in the gauge length and the clamps $\left(\tau_{F, G L}=500 \mathrm{kPa}, \tau_{F, \text { grips }}=5 \mathrm{MPa}\right)$ rather than the position, simply the number of unbroken layers in the gauge length determines the stress reached. 


\section{$5 \quad$ Particulate deposits}
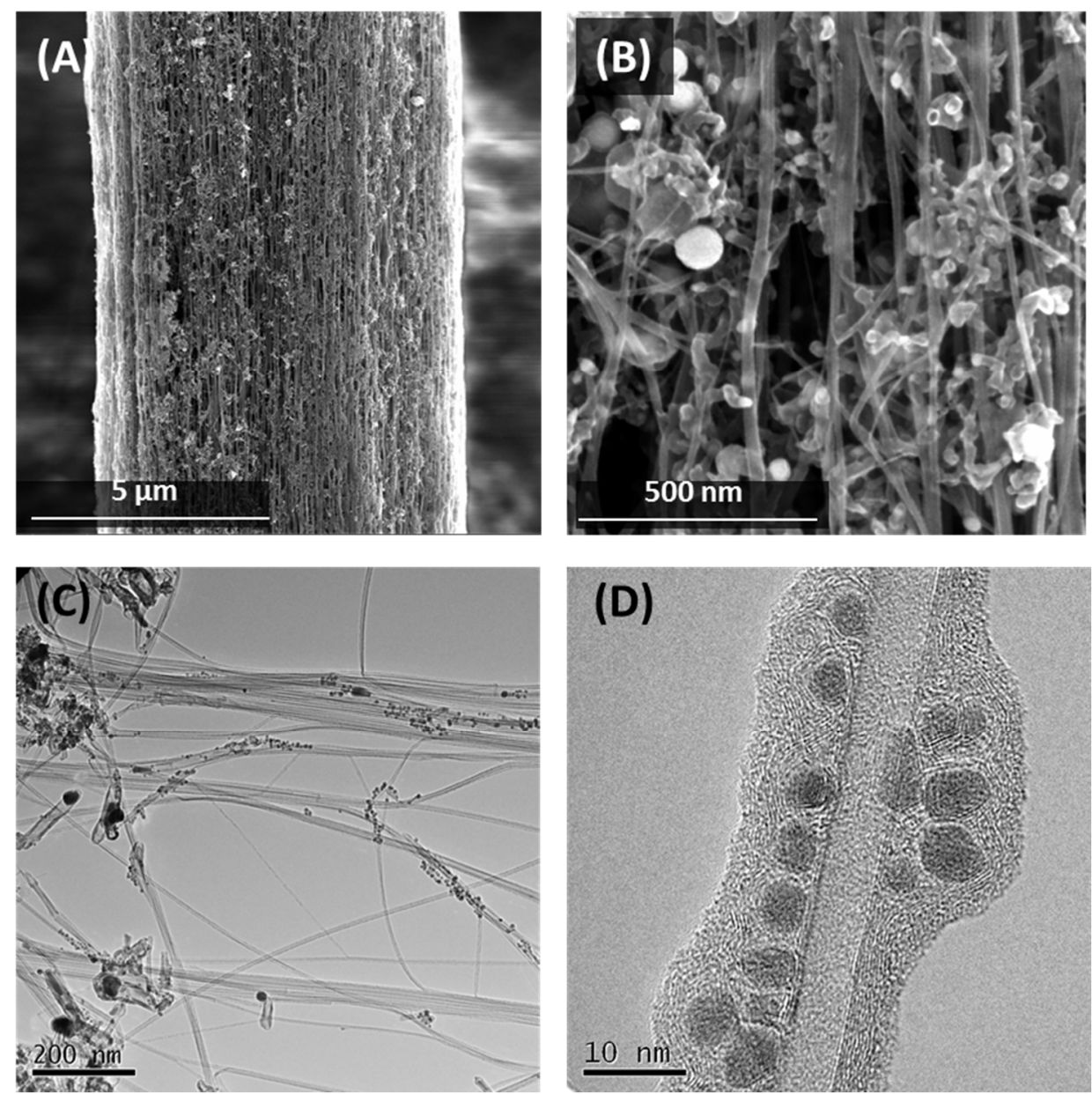

Figure S 5: $A+B$ ) Scanning electron micrographs of high strength fibre sample [17] showing exceptional amount of impurities of all kinds identified. C) TEM of bundles of Type Al fibre, showing left some deformed short MWCNTs responsible for the several $\mu \mathrm{m}$ big cluster impurities [22] and grape like clusters of unused catalyst residues close to the bundles, coated in carbonaceous material (enlarged in D) 


\section{$6 \quad$ Experimental short gauge length tests}

Figure S 6 shows long and short gauge length tests for three different fibre samples: 2 MWCNT samples Type A (spun from methane, thiophene and ferrocene) impure (A1) and pure (A2), and a predominantly SWCNT sample Type B (toluene, thiophene and ferrocene), pure. The detailed description of Type A and Type B can be found in [22]. Here, the term impurity relates primarily to the presence of large clusters of deformed MWCNTs, whose synthesis can be prevented by an increased carrier gas flow rate during spinning, and to unused catalyst particle entrapment, which was also significantly reduced (reduction of iron residue by factor 10). However, as the precursors for Type A1 and A2 are the same, the formation of the loadbearing CNTs and the bundle coating material is equivalent.

Type A1
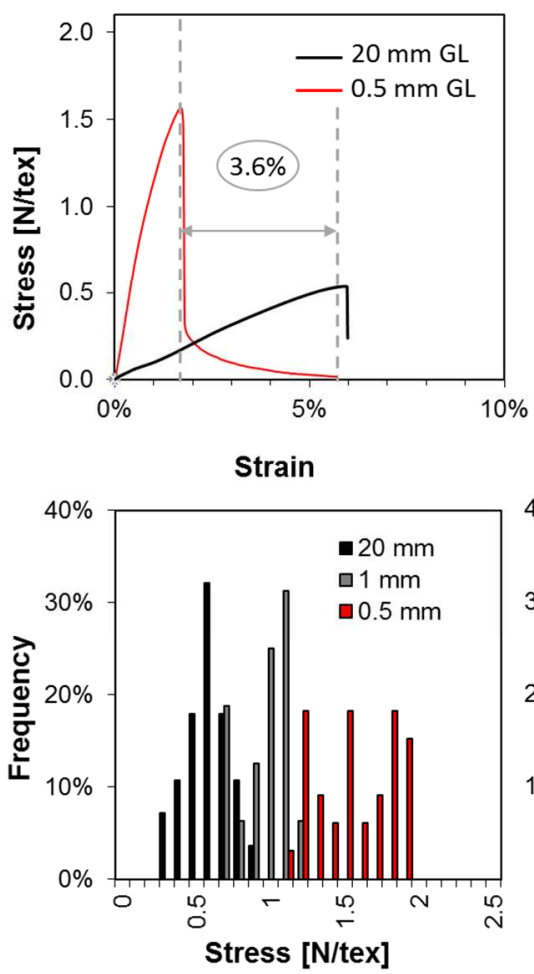

Type A2
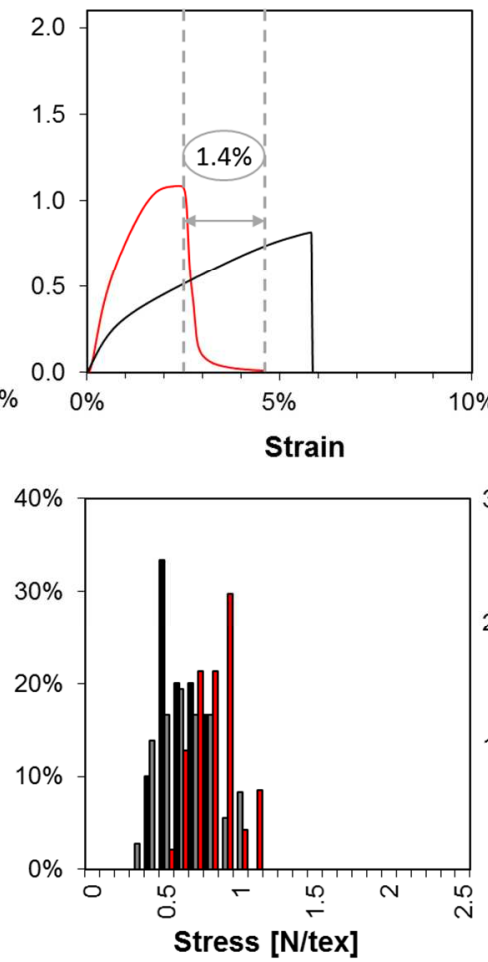

Type B

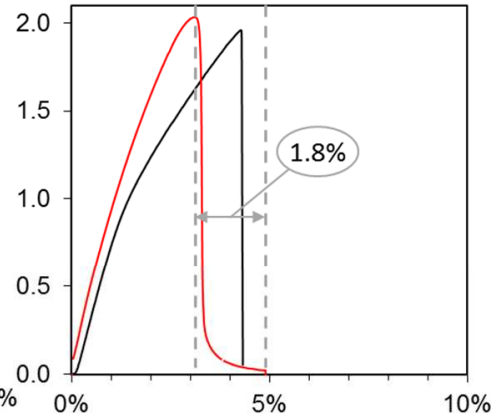

Strain

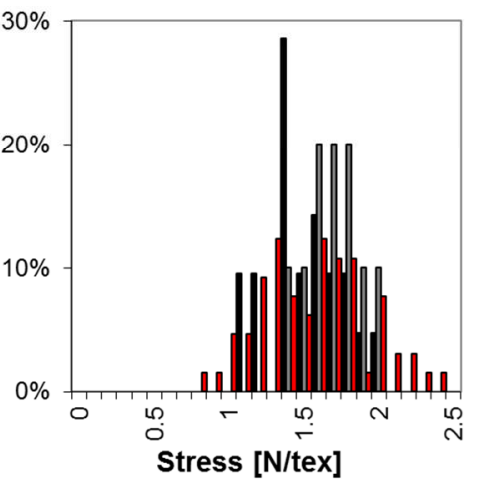

Figure S 6: Stress-strain curves (top row) and statistical stress evaluation (bottom row) of impure MWCNT fibre (Type A1, left), pure MWCNT fibre (Type A2, middle) and pure SWCNT fibre (Type B, right). The short gauge length tests have been corrected for strain deformation in the grips. Impure fibre (A1) shows an increased strength the shorter the gauge length, and a long elongation tail. The combination of those features led to the interpretation that the short gauge length is in the range of the CNT length. Type B shows overall a higher strength, which we attribute to a high amount of bundle coating, but the typical short gauge length strength increase fails to appear. 
For impure fibre Type A1 we observed the typical behaviour of staple yarns, showing higher strength for shorter gauge length, particularly when the gauge length gets close to the staple length. We can distinguish two domains in the breakdown. The first domain is due to the stretching of bundles around impurities, if present, and the aligning of the bundles parallel to the fibre axis. The second domain is due to the breaking of inter-bundle forces, sliding between or inside the bundles, and plastic deformation up to the breakdown. After the main breakdown, we observe an additional elongation tail at short gauge lengths which can be attributed to the overlap of last bridging bundles, after the majority of bundles in the fibre has slid out of contact with their neighbours. This elongation tail is not observed for long gauge length as the standard test speed was $10 \% \mathrm{GL} / \mathrm{min}$ and is too fast to resolve the elongation tail of a few micron displacement. Upon prevention of big cluster formation, the fibre structure was significantly improved and the performance more consistent, however, the ultimate tensile strength was not increased [22], and the strength is very consistent for all gauge lengths. Type B, spun from different precursors and showing a different type of CNTs but above all a significantly higher amount of co-synthesised bundle coating, shows overall twice the strength, but no further strength increase at short gauge length.

The after-break-elongation is about halved from Type A1 (3.6\%) to Type A2 (1.4\%), and Type B (1.8\%). It reasonable to assume, that this reduced elongation indicates a decreased CNT or bundle length, due to higher carrier gas flow which reduced the residence time of the CNTs in the reaction zone. For Type A2 and B, we conclude that the gauge length is still sufficiently large relative to the CNT length, so that we do not yet see the yarn like strength increase for short gauge length. For Type B for $1 \mathrm{~mm}$ and $0.5 \mathrm{~mm}$ the tensile strength is steadily increasing as bundles are actually drawn out of the grips. Hence, the short gauge length test provides, if indirectly, an estimation of the CNT length, otherwise almost impossible to analyse in bulk. 